\title{
Self-Aware Machine Learning for Multimodal Workload Monitoring During Manual Labor on Edge Wearable Sensors
}

\author{
Giulio Masinelli ${ }^{\circledR 1}$, Farnaz Forooghifar ${ }^{\circledR 1}$, Adriana Arza ${ }^{\circledR 1}$, \\ Amir Aminifar ${ }^{\circledR 1,2}$, and David Atienza ${ }^{\circledR 1}$, \\ ${ }^{1}$ École Polytechnique Fédérale de Lausanne (EPFL), Lausanne, Switzerland. ${ }^{2}$ Uppsala University, Uppsala, Sweden
}

\begin{abstract}
The design of reliable wearable technologies for real-time and long-term monitoring presents a major challenge. Self-awareness is a promising solution that enables the system to monitor itself in interaction with the environment and to manage its resources more efficiently. In this work, we aim to utilize the notion of self-awareness to improve the battery life of edge wearable sensors for multimodal health and workload monitoring. Specifically, we consider cognitive workload detection during manual labor as a case study to illustrate the impact of our proposed technique in wearable technologies. Our multimodal machine-learning algorithm is able to detect cognitive workload during manual labor with a performance of $81.75 \%$. By adopting the notion of self-awareness, we achieve an improvement of $27.6 \%$ in energy consumption, with less than $6 \%$ of performance loss.
\end{abstract}

Index Terms — Self-awareness, Machine learning, Workload monitoring, Manual labor, Multimodal, Edge wearable systems.

\section{INTRODUCTION}

$\mathbf{S}$ ELF-AWARENESS is the property of a system to evaluate its self-performance in the interaction with the environment and to take corrective measures based on such feedback to maximize the performance [1]-[3]. In such self-aware systems, several parameters of the system and the environment are observed to take the appropriate actions and to meet the requirements, while optimizing the objectives. Edge wearable devices based on multimodal sensors can also utilize the concept of self-awareness to improve their battery lifetime for long-term monitoring. Specifically, it allows to dynamically employ a reduced number of sensors whenever they can provide high confidence information and to switch to the full sensor mode using a high-dimensional feature set only when it is required [4], [5].

Wearable sensors are widely used for monitoring health and wellness. One emerging application for wearable technologies is daily monitoring of work-related stress including cognitive workload. Indeed, high cognitive workload affects workers' performance and, therefore, its monitoring can help to improve working conditions and, as a result, the productivity/efficiency of the workers itself. This is especially important during manual labor, where the workers have to follow a specific procedure with time and quality constraints. Specifically, there is the need of understanding how the worker's cognitive workload relates to the specific procedure's step they are accomplishing and how the overall methodology can be improved in a way that reduces the overload sources and work accidents/injuries by providing timely feedback.

To enable the monitoring of cognitive workload during manual labor, the system has to be fully wearable, minimally obtrusive, and agnostic to postural changes and physical activities that may trigger some physiological changes. Although it has been demonstrated that cognitive workload can be detected by measuring non-invasive physiological signals [6]

Manuscript received Month day, Year; revised Month day, Year. Corresponding author: Giulio Masinelli, E-mail address: giulio.masinelli@epfl.ch. and employing machine-learning techniques [7], [8], to the best of our knowledge, workload detection during manual labor has not been previously addressed in the literature.

Stress and cognitive workload are by nature multimodal and a single biomarker cannot comprehensively evaluate their response [9]. In general, workload triggers alterations in the sympathetic and parasympathetic balance, provoke hormonal responses, behavioral changes and/or decrease cognitive skills [10]. Therefore, given the current accessibility of wearable devices, a real-time monitoring system of cognitive workload is now possible by collecting the biosignals that represent the previously mentioned alterations: photoplethysmography (PPG), skin temperature (SKT), respiration (RSP), electrocardiogram (ECG) and electrodermal activity (EDA) [6]. Several previous studies [11], [12] demonstrate the possibility of offline workload detection based on such physiological signals and advanced machine-learning techniques, including eXtreme Gradient Boosting and Random Forest. However, wearable technologies are often extremely limited in terms of available resources, including battery lifetime, which makes the usage of such complex multimodal machine-learning techniques challenging.

In this article, we adopt the notion of self-awareness for dynamic energy-management on mobile and wearable platforms and propose a self-aware machine-learning technique to improve the lifetime of such systems, without any major performance loss. Our proposed technique is particularly suited for multimodal monitoring settings with several physiological signals, due to the complexity and energy overhead of such systems. Specifically, we evaluate our proposed machine-learning technique for multimodal cognitive workload monitoring during manual labor and demonstrate its effectiveness experimentally.

\section{Multimodal Workload Monitoring}

Cognitive workload can be defined as the physical or mental requirements associated with a task or combination of tasks [13]. On one hand, physical workload is the measurable portion 
of physical resources expended when performing a given task and it is affected by a range of factors (such as nature of work, training, motivation and environmental factors). On the other hand, cognitive workload reflects the mental strain resulting from performing a task under specific environmental and operational conditions, considering the capabilities of the operator to respond to those demands [10].

A multimodal monitoring system for cognitive workload assessment using physiological signals can be conceptually divided into three mains steps, namely, signal acquisition, preprocessing, and workload detection. The signal acquisition refers to the measurement of the biosignals proposed in our multimodal monitoring technique, such as PPG, SKT, RSP, ECG, and EDA. Then, in the prepossessing, each signal is filtered and different biomarkers are extracted. Figure 1 shows the main biomarkers obtained from each time series and are computed as in [9] and [12]. Next, from those biomarkers, several features in time and frequency domain are extracted, as described in our previous work [12]. Finally, a reduced set of the most important features is used for workload detection. Specifically, we adopt the Random Forest algorithm for both feature selection and classification, a robust state-ofthe-art classification algorithm that uses the so-called bagging technique to avoid overfitting. Bagging stands for Bootstrap AGGregation and aims at combining multiple Decision Trees so that the final label of a new data sample is determined by majority voting among all the trees.

In the context of manual labor, physical activity and different body positions are part of the detection problem since they trigger some physiological changes. Thus, we need to select the features that correlate more with cognitive workload and less with physical activity and body positions. Therefore, for the preliminary exploration, we obtain a total of 125 features: 15 from ECG, 44 from RSP, 59 from PPG, 5 from EDA and 2 from SKT.

However, a high number of features makes the model complex and, as a result, less energy efficient as well as more prone to errors due to the higher variance. Consequently, there is the need for a feature selection technique to considerably reduce the number of features.

We adopt the feature selection technique described in [12] and [11]. To be specific, the first stage of feature selection concerns the removal of the features that are not statistically relevant in distinguishing the experimental phases described later in IV-A. Then, among the features that show a Pearson's correlations coefficient higher than 0.99, we select the most important one. Finally, we apply the Recursive Feature Elimination technique (RFE) to select the most informative features for cognitive workload detection during manual labor.

RFE exploits the property of tree-based machine learning algorithms of performing feature selection during their training by ranking the features by importance. Specifically, after training the Random Forest classifier on the training set using the leave-one-subject-out approach, for every feature, a score positively correlated to its importance is provided. The model is then retrained using a smaller feature set obtained by discarding the least important feature. The procedure is recursively repeated to obtain the accuracy in cross validation,

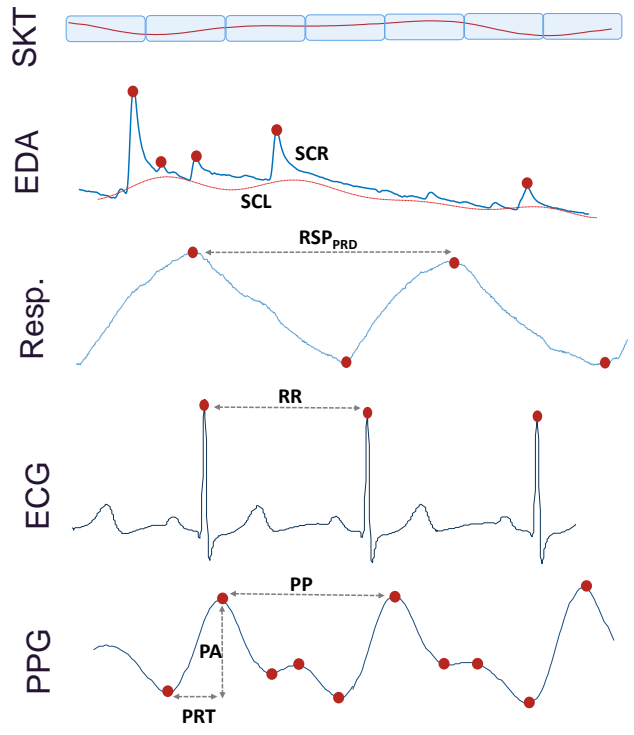

Figure 1: Biomarkers extracted from skin temperature (SKT), electrodermal activity (EDA), respiration (RSP), electrocardiogram (ECG) and photoplethysmography (PPG) signals.

as well as the feature set used in every iteration. Lastly, the final feature set is chosen to be one of the ones that have the highest cross-validation accuracy and a reduced feature count.

As seen, the feature selection technique allows us to identify the best set of features, which is particularly useful for the deployment of the model on embedded platforms. In fact, it substantially reduces the cardinality of the feature set, without affecting the classification performance. However, due to the multimodality of the workload, a further reduction of the feature set dimensionality is not possible without the degradation of the detection capabilities. This is where the concept of selfawareness comes into play, which allows using a reduced number of sensors, and consequently, a simple model, whenever it can provide a high level of confidence, for example, when the subject is at rest. In this case, in fact, it is sufficient to acquire a few signals to determine the state of the user. When, however, it is necessary to have a greater capacity of discernment, for example, to distinguish between cognitive workload and physical workload, the system is able to switch to the full mode using the high dimensional features extracted after turning on all the available sensors. This concept is further explained in the following section.

\section{Self-Aware Machine Learning on Edge}

As stated, workload monitoring requires a multimodal analysis, i.e., the acquisition of multiple signals and the execution of several feature extraction algorithms, which comes with the overhead of a short battery lifetime for the wearable monitoring device compared to a single sensor device and simple processing. On the other hand, as we'll see in Section $\mathrm{V}-\mathrm{B}$, a small subset of the biosignals is often sufficient for a confident classification of the subject's status. As a result, we 


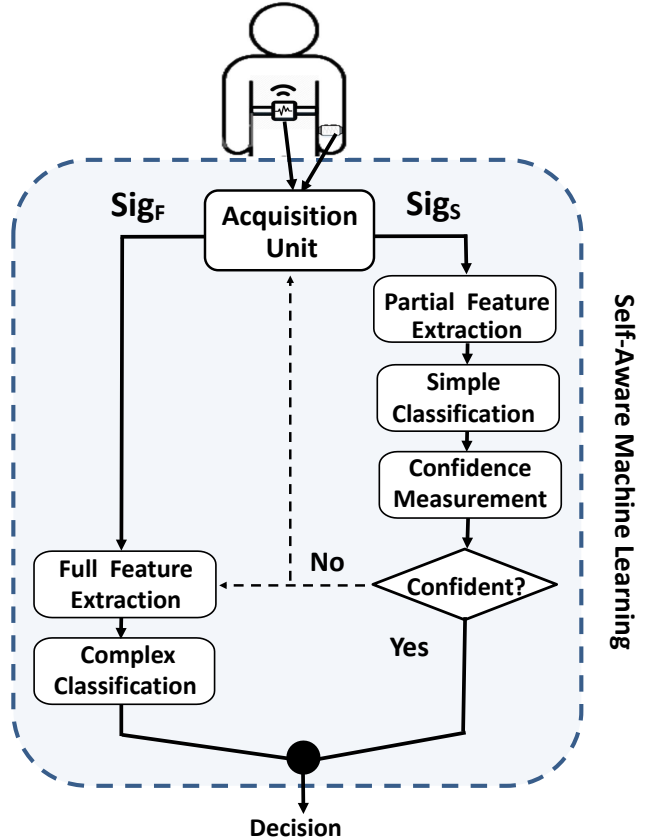

Figure 2: Overview of our self-aware classification algorithm for cognitive workload monitoring.

can use a partial subset of signals and features with low energy overhead while the others are inactive by default, and only move to the full-system acquisition and processing when all the features are required to provide a more reliable detection of the subject's status. This approach enables our system to maintain its high performance while significantly reducing the energy consumption.

Moreover, other methods can be considered as adopting the general concept of self-awareness, such as [14] and [15], which adapt the number of stages the input signal goes through depending on the input sample. Our system differs from these works in the way that it 'reacts' to different samples, not just by dynamically adjusting the computation complexity, but also by requiring a more comprehensive description of the sample by switching on a new set of sensors when required.

The high-level overview of our proposed self-aware detection system is shown in Figure 2. The acquisition unit consists of different sensors worn by the workers during their manual labor. Initially, only a subset of sensors is active to acquire the small subset of signals $\left(\mathrm{Sig}_{S}\right)$, extract their features and make an inference/decision with the simple classifier. Then, based on the confidence measurement, if the outcome is reliable enough, the final decision is provided by the partial system mode. Otherwise, we switch to the full system mode, where all the sensors are activated to acquire all the signals $\left(S i g_{F}\right)$. In this case, the full feature extraction and the complex classification are also turned on to make the final decision.

As discussed before, Random Forest is the classification model that is used to obtain the final decision on the workload from the selected features. In order to measure the confidence of the partial system mode, we consider a threshold on the number of trees that must agree on the classification result of the Random Forest classifier. If the number of trees is less than this threshold, we consider the classification as not reliable enough and we switch to the full system mode to ensure the trustworthiness of the classification decision.

In order to determine the value of the optimal threshold, we have analyzed the classification performance in crossvalidation to varying of the threshold (using the leave-onesubject out approach in the training set). The optimal one is chosen to be the lowest threshold that does not decrease the classification accuracy. Let's now estimate the complexity and energy consumption of our proposed self-aware classification algorithm. The partial system mode is invoked for every decision. However, the full-system mode is activated only with probability $1-P$, where $P$ is the probability of having a reliable outcome using the partial system mode (which depends on the problem and dataset). Therefore, the total energy consumption of our wearable system is as follows:

$$
E_{\text {total }}=E_{\text {partial }}+E_{\text {confidence }}+(1-P) \cdot E_{\text {full }},
$$

where $E_{\text {partial }}, E_{\text {confidence }}$ and $E_{\text {full }}$ are the energy consumption of the partial system mode, the confidence measurement and the full system mode, respectively. $E_{\text {partial }}$ is defined as:

$$
E_{\text {partial }}=\sum_{i=1}^{M}\left(E_{A C(i)}+E_{F E(i)}\right)+E_{M L_{\text {partial }}},
$$

where we assume that the first $M$ signals are used in the partial system mode, $E_{A C(i)}$ and $E_{F E(i)}$ are the acquisition and feature extraction energy for the $i^{\text {th }}$ sensor, and $E_{M L_{\text {partial }}}$ is the energy overhead of the machine-learning technique for the simple model. The energy overhead of the full system mode $\left(E_{f u l l}\right)$ is obtained similarly as follows:

$$
E_{\text {full }}=\sum_{i=M+1}^{N}\left(E_{A C(i)}+E_{F E(i)}\right)+E_{M L_{f u l l}},
$$

where $N$ is the total number of signals and $E_{M L_{f u l l}}$ is the energy overhead of the machine-learning technique for the full model. Note that the first $M$ signals are already taken into account in Equation 2.

\section{EXPERIMENTAL SETUP}

To evaluate the feasibility of our proposed technique, we use experimental data for building the workload detection model and a multi-sensor platform with an ultra-low power microcontroller unit for the estimation of the energy consumption.

\section{A. Experiment for Workload Detection During Manual Labor}

As stated, there are no previous studies nor databases that address the problem of workload detection during manual

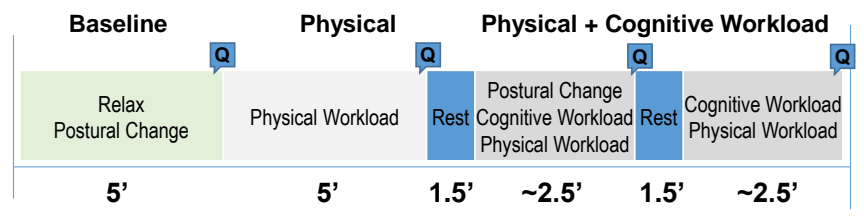

Figure 3: Protocol of the experiment. 
labor. Therefore, we have designed an experimental protocol to induce a moderate workload on the participants by performing some of the most common tasks among manual workers (Ethical approval HREC:037-2019). In total, we have collected biosignals from 18 young and healthy subjects.

During manual labor, worker's physical activity and body position trigger changes on the biomarkers, so special care has to be taken in order to not confuse those changes with the physiological body response of stress.

Therefore, the research protocol's design includes different states i.e., no-workload plus different body positions and a workload state, comprising two stages including moderate physical working activities and a combination of cognitive and physical workload. Figure 3 summarizes the experimental protocol.

Initially, in the baseline phase, a relaxing audio is reproduced to induce a relaxed state, while adopting five different positions during one minute each one (i.e., standing arms by the side, standing with arms raised, squatting, walking and sitting). Next, the phase of physical workload, which consists in tightening 12 screws in three blocks of wood located at different heights (at forehead height, at torso height and on the floor). Finally, the last phase consisting in the combination of the two previous stages and cognitive workload. The latter consists of solving several arithmetic tasks while tightening the screws with a timer that alarms the subject in case he/she is taking too long.

The rest periods are meant to allow the biosignal to stabilize to the normal state after each physical activity stage. Between contiguous stages of the experiment, a self-report questionnaire was completed by the subject. The questionnaire (Q) consists of a visual analogue scale in which the subjects can select their stress level from 0 to 100 at that moment. This questionnaire has been used for the evaluation of the effectiveness of the experiment protocol design, namely, to verify that the workload phases are correctly perceived as more stressful than the relaxing one. Table I shows the summary of the participants' reported stress levels for each stage.

Table I: Results from the subjects' self-evaluation of the different phases of the experiment

\begin{tabular}{|c|c|c|c|c|}
\hline Stage & Mean & Min & Max & Std \\
\hline Relax & 5.11 & 0 & 18 & 4.85 \\
\hline Phy & 23.53 & 7 & 50 & 12.39 \\
\hline Phy + Cogn & 71.05 & 30 & 89 & 12.57 \\
\hline
\end{tabular}

\section{B. Model Learning and Performance Evaluation}

Since we are aiming at designing a system that is agnostic to postural changes, we will label the three phases of the experiment in the following way: Baseline $\rightarrow 0$, Physical $\rightarrow 1$, Physical + Cognitive Workload $\rightarrow 2$. In this way, since postural change is common among most of the phases, the algorithm should be able to reject its influence due to the irrelevance in the classification. All the signals are segmented in windows of one-minute with an overlap of 30 seconds [11]. The dataset obtained is made up of 456 samples, i.e., the number of 1minute windows extracted $\in \mathbb{R}^{125}$ (as the number of features).
To ensure generalization of our results, the collected dataset is randomly split into training and test sets, containing $80 \%$ and $20 \%$ of the data, i.e. 14 and 4 subjects, respectively. During the training of the model, we always used leave-one-subject-out cross-validation scheme on the training set. Specifically, the number of folds for cross-validation is equal to the number of subjects in the training set. In this way, each fold contains only the data of a single subject to avoid the overlapping of data between the training and validation sets.

The performance of the proposed algorithm and model are evaluated by measuring the specificity $(S p e c)$, sensitivity (Sen), and the geometric mean (gmean) of these two metrics. The geometric mean gmean is adopted since its high value reflects the fact that both specificity (Spec) and sensitivity (Sen) are high in value, which is equal to have high quality detection. Conversely, if the geometric mean gmean is low, it means that Spec, Sen, or both are low, which is undesirable. To be specific, since we are considering a ternary classification problem, we evaluate the overall specificity and sensitivity as the gmean of those measures per class (one against all approach).

\section{Evaluation Hardware Platform}

To evaluate the system performance in terms of energy consumption, we assume to adopt a single-chip system-on-chip platform, where the analog front-end design is derived from [16] and the microcontroller is an ARM Cortex M3. The energy consumption for signal acquisition is assumed to be the same as reported in the measurements from [16], and it is presented in Table II.

Table II: Signals acquisition specifications

\begin{tabular}{|c|c|c|c|}
\hline Signal & Fs $(\mathrm{Hz})$ & Energy $(\mathrm{mJ}) *$ & Decimation factor \\
\hline EDA & 64 & 2.34 & 16 \\
\hline PPG & 128 & 16.02 & 2 \\
\hline RSP & 128 & 9.9 & 2 \\
\hline SKT & 4 & 0.54 & 1 \\
\hline
\end{tabular}

* per 60-seconds window, from [16]

To measure the energy consumption of the different algorithms for feature extraction and inference, we use the Simplicity Studio software energy profiler on a Cortex-M3 based EFM32LG-STK3600 board, compose of a EFM32LG TM Leopard Gecko 32-bit microcontroller unit (MCU) with $3 \mathrm{~V}$ supply, $256 \mathrm{kB}$ flash memory and $32 \mathrm{kB}$ RAM. Before processing any of the aforementioned signals, they are downsampled via software by the decimation factor reported in Table II. The decimation allows reducing the energy overhead of the feature extraction algorithms.

\section{EXPERIMENTAL EVALUATION}

In this section, we evaluate our proposed self-aware machinelearning methodology for multimodal cognitive-workload monitoring during manual labor, in terms of detection performance as well as energy consumption. 
Table III: Estimated Energy consumption per component in 60 -seconds windows

\begin{tabular}{|l|c|}
\hline Component & Energy (mJ) \\
\hline EDA: $S C R_{\text {Power }}, S C L_{\text {mean }}$ & 3.85 \\
\hline SKT: $S K T_{\text {Power }}$ & 0.55 \\
\hline PPG: $P D T_{\text {mean }}, P A_{\text {mean }}, P A r_{S T D}$, & 360.97 \\
$P D T_{P_{[0.04-0.15 H z]}, P W_{\text {mean }}, P R T_{\text {Power }}}$ & \\
\hline $\begin{array}{l}\text { RSP: } P_{[0.5-0.75 H z]}, \text { Rate }_{\text {mean }}, P r d_{\text {mean }} \\
\text { Ratio }\end{array}$ & 12.45 \\
\hline Simple model evaluation & $0.05 \mathrm{H}[\mathrm{P}]$ \\
\hline Confidence evaluation & 0.005 \\
\hline Full model evaluation & 0.82 \\
\hline
\end{tabular}

SCR: Skin Conductance Response, SCL: Skin Conductance level, PDT: Pulse Decreasing Time, PA: Pulse Amplitude, PAr: Pulse Amplitude of reflective wave, PW: Pulse Width, PRT: Pulse Rising Time, Prd: Period, $P_{\left[x_{1}-x_{2}\right]}$ : power in the defined frequency band, Power: total power.

\section{A. Multimodal Workload Monitoring}

Firstly, we evaluate the proposed workload detection methodology and demonstrate the models' generalization abilities by testing them on an unseen test set.

As described in Section II, by identifying the meaningful features, we reduce the size of the feature set from 125 to 81 . Next, using RFE we select a set of the 13 most significant features.

A reduced feature set also implies a reduction in power consumption, since we only measure and process the biosignals that are informative. In this case, the biosignals that need to be monitored are PPG, RSP, EDA and SKT and the used feature set is reported in Table III. The energy consumption for 60seconds window of the workload detection algorithm per main component is reported in Table III. The reported energy for each signal component includes both sensor and feature extraction algorithms.

Finally, the full model performance evaluated on an unseen test set is: $92.02 \%, 82.04 \%$ and $86.89 \%$ for specificity, sensitivity and gmean, respectively. This means that the proposed multimodal machine-learning algorithm, based on Random Forest, is able to distinguish among the baseline phase, the intense cognitive phase and the physical phase. Our model outperform the current state-of-the-art accuracy reported in [11] and [12] (86\% and $84.13 \%$, respectively) considering a ternary classification problem instead of a binary classification (i.e., stress vs. no stress), and also a harder problem due to high noise level caused by the additional physical activity. In addition, the relatively low complexity of the algorithm, employing just 13 features from PPG, RSP, EDA and SKT signals, makes it suitable for an implementation on a resource-constrained embedded system.

The combination of multiple biosignals and features allows the model to reach high accuracy. Even though, we can obtain similar results, with the additional advantage of significant energy reduction, by introducing self-awareness to the system.

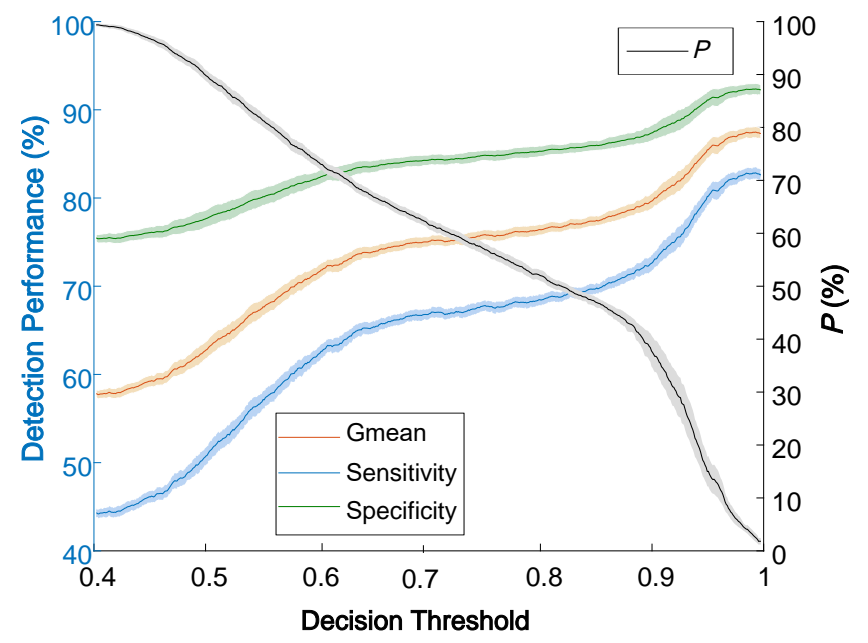

Figure 4: Workload monitoring system's performance and probability of using the simple model vs confidence threshold.

\section{B. Self-Aware Multimodal Workload Monitoring}

The first step to apply the concept of self-awareness, presented in Section III, is to evaluate the energy consumption of every signal and its contribution to the person's state detection. In particular, we identify the feature extracted from EDA and SKT as the least power-hungry features considering both the acquisition energy $(2.34 \mathrm{~mJ}$ for EDA and $0.54 \mathrm{~mJ}$ for SKT, see Table II) and the energy expended for processing (1.52 $\mathrm{mJ}$, see Table III). Additionally, the EDA represents a direct activation of the sympathetic system and it is triggered by both physical activity and workload. Similarly, the SKT correlates with vasoconstriction variations and compensates for the effect of ambient temperature on the EDA. Therefore, we assume that the partial set of features is only based on EDA and SKT signals. The decision threshold for our self-aware system has been fixed to $92 \%$ by looking at both the energy consumption reduction and the performance of the model in cross validation on the training set.

Figure 4 shows the performance evaluated on the test set of the self-aware system and the probability of having a reliable outcome using the classifier of the partial system mode, plotted with respect to the confidence threshold. As this threshold increases, the use of the full-system mode e.g., full feature set and complex classifier also increase and, as a result, the accuracy is boosted. We notice a significant gap between the sensitivity and specificity, which is caused by the fact we are considering a ternary classification problem. Assuming a balanced test set, the sensitivity and specificity of a random ternary classifier are $\frac{1}{3}$ and $\frac{2}{3}$, respectively.

Figure 5 shows the energy consumption of the self-aware system and the performance (gmean), plotted with respect to the confidence threshold. As before, as the threshold increases, the energy consumption rises, since the number of time that the full-system mode is activated is higher. This, in turn, leads to higher energy consumption and classification performance.

The performance and energy consumption of the systems with and without self-awareness are shown in Table IV. The energy consumption of the low-power system mode, which 
Table IV: Comparison between our workload detection system with and without self-awareness

\begin{tabular}{|c|c|c|c|c|c|c|c|}
\hline Mode & Decision th (\%) & $P(\%)$ & CV-Acc (\%) & Gmean (\%) & Sens (\%) & Spec (\%) & Energy (mJ) \\
\hline Partial system & - & 100 & 56.07 & 57.78 & 44.25 & 75.46 & 4.66 \\
\hline \multirow{4}{*}{ Self-aware system } & 50 & 89.68 & 59.92 & 63.01 & 51.18 & 77.58 & 43.28 \\
\cline { 2 - 8 } & 60 & 73.88 & 67.04 & 71.19 & 61.77 & 82.07 & 102.4 \\
\cline { 2 - 8 } & 70 & 61.64 & 71.65 & 75.23 & 67.04 & 84.42 & 148.2 \\
\cline { 2 - 8 } & 80 & 52.52 & 76.73 & 76.46 & 68.52 & 85.33 & 182.3 \\
\cline { 2 - 8 } & 90 & 37.40 & 77.98 & 79.61 & 72.54 & 87.36 & 238.9 \\
\cline { 2 - 8 } & $\mathbf{9 2}$ & $\mathbf{3 1 . 5 2}$ & $\mathbf{7 8 . 5 9}$ & $\mathbf{8 1 . 7 5}$ & $\mathbf{7 5 . 4 8}$ & $\mathbf{8 8 . 5 6}$ & $\mathbf{2 7 0 . 8}$ \\
\cline { 2 - 8 } & 95 & 14.08 & 78.57 & 85.74 & 80.54 & 91.28 & 326.2 \\
\hline Full system & - & 0 & 78.36 & 86.89 & 82.04 & 92.02 & 374.24 \\
\hline
\end{tabular}

Decision th: Decision threshold, $P$ : Probability of using the partial system mode, Gmean: Geometric mean, Sens: Sensitivity, Specs: Specificity, CV-Acc: Average accuracy using cross validation on the training set, Energy: Energy consumption in 60-seconds windows.

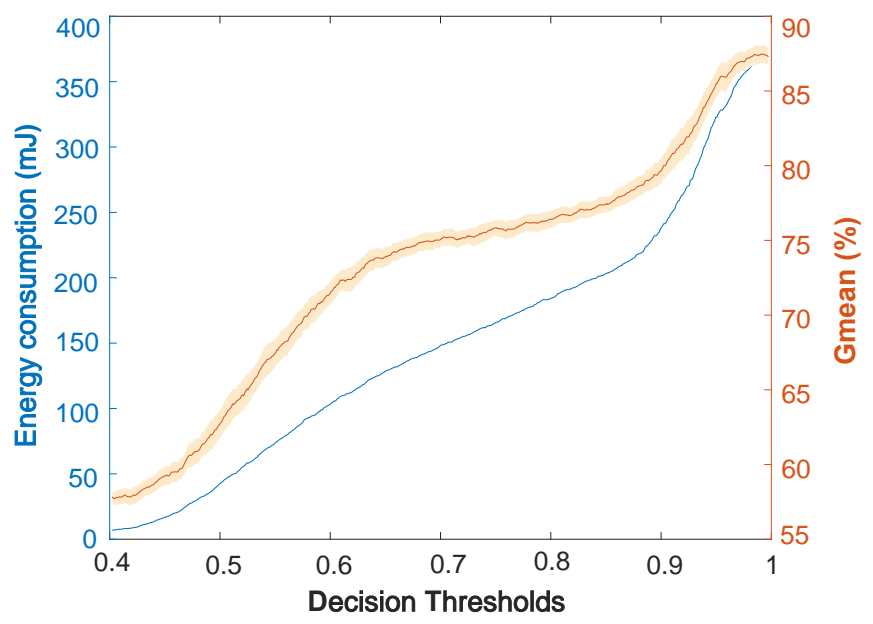

Figure 5: Energy consumption and classification performance versus confidence threshold.

only acquires and calculates three features from EDA and SKT signals, is just $1.25 \%$ of the energy required by the full system. Thus, in Equation (1), the dominant term is the last one, which is reduced with a factor of $1-P$ thanks to our self-aware technique. We observe that by increasing the decision threshold from $50 \%$ to $70 \%$ to $90 \%$ the energy consumption is reduced by $88.44 \%, 60.40 \%$, and $36.16 \%$, while the gmean is decreased by $27.48 \%, 13.42 \%$, and $8.38 \%$. This confidence threshold can be used by the designer to make a trade-off between the classification performance and the lifetime of the system.

\section{CONCLUSION}

In this article, we have introduced the notion of selfawareness for health and activity monitoring systems with multimodal machine-learning algorithms. In addition, we have shown the feasibility of cognitive workload detection during manual labor, which, to the best of our knowledge, has not been addressed before in the literature. In particular, we propose a multimode system, with a low-power mode that uses a subset of biosignals and a full-system mode, which is only activated if it is required to reach the desired reliability. Our proposed selfaware machine-learning algorithm is able to distinguish among baseline, cognitive workload and physical work with an $81.75 \%$ of gmean. The model has proved to perform similarly to the state of art accuracy ( [11], [12], 86\% and $84.13 \%$, respectively) considering a harder problem due to the additional physical activity and being a ternary classification problem instead of a binary classification (stress versus no stress).

Moreover, the proposed two-mode system improves the energy consumption by $27.6 \%$, without any major loss in classification performance.

\section{ACKNOWLEDGMENT}

This work has been partially supported by the MyPreHealth (grant no. 16073) project funded by Hasler Stiftung and by a Research Agreement of Schindler-Invention AG to EPFL (No. 2019-0001).

\section{REFERENCES}

[1] S. Kounev, J. O. Kephart, A. Milenkoski, and X. Zhu, Self-Aware Computing Systems. Springer Publishing Company, Incorporated, 1st ed., 2017.

[2] N. Dutt, A. Jantsch, and S. Sarma, "Toward smart embedded systems: A self-aware system-on-chip (soc) perspective," ACM Transactions on Embedded Computing Systems (TECS), vol. 15, no. 2, p. 22, 2016.

[3] M. Maggio, T. Abdelzaher, L. Esterle, H. Giese, J. O. Kephart, O. J. Mengshoel, A. V. Papadopoulos, A. Robertsson, and K. Wolter, "Selfadaptation for individual self-aware computing systems," in Self-Aware Computing Systems, pp. 375-399, Springer, 2017.

[4] F. Forooghifar, A. Aminifar, L. Cammoun, I. Wisniewski, C. Ciumas, P. Ryvlin, and D. Atienza, "A self-aware epilepsy monitoring system for real-time epileptic seizure detection," in ACM/Springer Mobile Networks and Applications (MONET), pp. 1-14, ACM/Springer, 2019.

[5] D. Pascual, A. Aminifar, and D. Atienza, "A self-learning methodology for epileptic seizure detection with minimally-supervised edge labeling," in 2019 Design, Automation \& Test in Europe Conference \& Exhibition (DATE), pp. 764-769, IEEE, 2019.

[6] M. Ranchet, J. C. Morgan, A. E. Akinwuntan, and H. Devos, "Cognitive workload across the spectrum of cognitive impairments: A systematic review of physiological measures," Neuroscience \& Biobehavioral Reviews, vol. 80, pp. 516-537, 2017.

[7] J. Heard, C. E. Harriott, and J. A. Adams, "A Survey of Workload Assessment Algorithms," IEEE Transactions on Human-Machine Systems, pp. 1-18, 2017.

[8] A. O. Akmandor and N. K. Jha, "Detecting and Alleviating Stress with SoDA," Computer, vol. 51, pp. 4-5, jul 2018.

[9] A. Arza, J. M. Garzón-Rey, J. Lázaro, E. Gil, R. Lopez-Anton, C. de la Camara, P. Laguna, R. Bailon, and J. Aguiló, "Measuring acute stress response through physiological signals: towards a quantitative assessment of stress," Medical \& Biological Engineering \& Computing, vol. 57, pp. 271-287, 12019. 
[10] B. Cain, "A Review of the Mental Workload Literature," Defence Research And Development Toronto (Canada), 2007.

[11] N. Momeni, F. I. T. Dell'Agnola, A. Arza Valdes, and D. Atienza Alonso, "Real-time cognitive workload monitoring based on machine learning using physiological signals in rescue missions," in International Engineering in Medicine and Biology Conference, no. CONF, 2019.

[12] V. Montesinos Canovas, F. I. T. Dell'Agnola, A. Arza Valdes, A. Aminifar, and D. Atienza Alonso, "Multi-modal acute stress recognition using offthe-shelf wearable devices," in International Engineering in Medicine and Biology Conference, no. CONF, 2019.

[13] S. Gudipati and A. Pennathur, "Physical and Mental Workload Assessment Techniques for Job Design," tech. rep.

[14] P. Panda, A. Sengupta, and K. Roy, "Conditional Deep Learning for Energy-Efficient and Enhanced Pattern Recognition," sep 2015.

[15] E. Park, D. Kim, S. Kim, Y. D. Kim, G. Kim, S. Yoon, and S. Yoo, "Big/little deep neural network for ultra low power inference," in 2015 International Conference on Hardware/Software Codesign and System Synthesis, CODES+ISSS 2015, pp. 124-132, Institute of Electrical and Electronics Engineers Inc., nov 2015.

[16] M. Konijnenburg, S. Stanzione, L. Yan, D. Jee, J. Pettine, R. van Wegberg, H. Kim, C. Van Liempd, R. Fish, J. Schuessler, H. de Groot, C. Van Hoof, R. F. Yazicioglu, and N. Van Helleputte, "A multi(bio)sensor acquisition system with integrated processor, power management, $8 \times 8$ led drivers, and simultaneously synchronized ecg, bio-z, gsr, and two ppg readouts," IEEE Journal of Solid-State Circuits, vol. 51, pp. 2584-2595, Nov 2016.

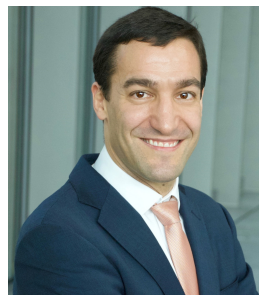

David Atienza david.atienza@epfl.ch

David Atienza is Professor of EE and Heads the Embedded Systems Laboratory (ESL) at EPFL. He received his $\mathrm{Ph} . \mathrm{D}$. in computer engineering from UCM and IMEC. His research focuses on design methodologies for Internet of Things (IoT) and edgeAI computing. He has published more than 300 papers and 10 patents.

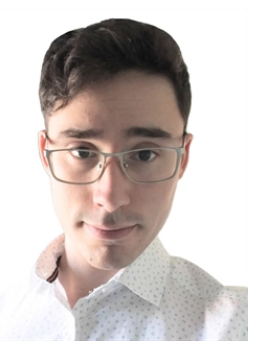

Giulio masinelli giulio.masinelli@epfl.ch

Giulio Masinelli is a Ph.D. researcher in the Embedded Systems Laboratory (ESL) at EPFL, Switzerland. $\mathrm{He}$ received his M.Sc. in Electrical Engineering (data science and IoT specialization) in 2019, at EPFL. His research interests include signal processing, machine learning, and IoT, with particular attention to low energy solutions.

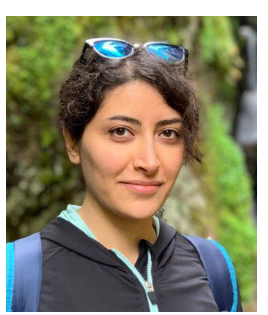

Farnaz Forooghifar farnaz.forooghifar@epfl.ch Farnaz Forooghifar is a Ph.D. researcher in the Embedded Systems Laboratory (ESL) at the Swiss Federal Institute of Technology Lausanne (EPFL), Switzerland. She received her M.Sc. in electrical engineering from University of Tehran, Iran, in 2017. Her research interests include real-time health monitoring systems, machine learning, and embedded systems design.

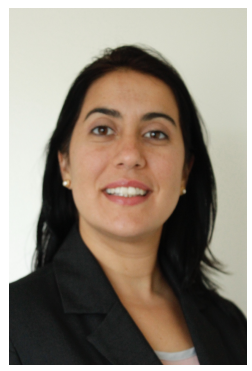

Adriana Arza adriana.arza@epfl.ch

Adriana Arza is a Post-Doctoral researcher in the Embedded Systems Laboratory at the EPFL, Switzerland. She received her Ph.D. degree in Microelectronic and Electronic Systems in 2017 from Autonomous University of Barcelona. Her research interests include energy-aware algorithms and system-level design of wearable sensors for personalized health and wellness applications.

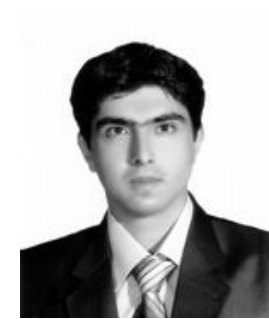

Amir Aminifar amiraminifar@it.uu.se

Amir Aminifar is an Assistant Professor in the Department of Information Technology at Uppsala University, Sweden. He received his Ph.D. degree from the Swedish National Computer Science Graduate School (CUGS), Linköping University, Sweden, in 2016. His research interests include mobile-health technologies and medical informatics. 